Año 9. Núm. 24 (Edición especial julio- diciembre 2016)

http://revistainvestigacionacademicasinfrontera.com

Revista de Investigación

Académica sin Frontera

ISSN: 2007-8870

\title{
Grado de Responsabilidad Social Empresarial (RSE) en las Empresas Industriales del Sur de Sonora
}

\author{
Modesto Barrón Wilson \\ María del rosario Zayas Campas \\ Leticia María González Velásquez
}

\section{Resumen}

En la actualidad es de vital importancia que las empresas se puedan sumar a los esfuerzos para mejorar la situación económica y social del lugar donde están insertas.

La Responsabilidad Social Empresarial (RSE), se puede definir como el compromiso que adquiere una empresa ante sus grupos de interés y la sociedad en general. Se trata de la integración voluntaria por parte de las empresas de las preocupaciones sociales y ambientales en sus operaciones comerciales y en las relaciones con sus interlocutores.

Todas las empresas deben cumplir con el código de ética de los negocios, respecto a la aplicación de la justicia y la equidad, para enfrentar situaciones que presentan conflictos éticos entre los empleados, clientes, proveedores o la sociedad en general.

La presente investigación es enfocada a las Medianas Empresas Industriales del sur de Sonora, con el propósito fundamental de conocer los Avances en la implementación del modelo de Responsabilidad social empresarial (RSE).

Así mismo, se trata también de identificar si su esquema organizacional corresponde al modelo de empresa socialmente responsable.

Posteriormente, los resultados nos muestran las áreas de oportunidad y todos los aspectos en las cuales las empresas industriales pueden mejorar estas prácticas de responsabilidad social.

Palabras clave: Responsabilidad, Empresa, Ambientales, implementación, Ética 
Año 9. Núm. 24 (Edición especial julio- diciembre 2016)

http://revistainvestigacionacademicasinfrontera.com

Revista de Investigación

Académica sin Frontera ISSN: 2007-8870

\section{Introducción}

Cuando se celebró la Cumbre de la Tierra en 1992, en Río de Janeiro, Brasil, los países ahí reunidos, tomaron acuerdos en los cuales cada país se comprometía a promover una serie de cambios tanto políticos como económicos y sociales, para reconocer que es importante la protección del medio ambiente, los derechos humanos, el desarrollo humano equilibrado y la paz.

El resultado de esa reunión cumbre, fue un documento al que llamaron Carta a la Tierra, en la que se establecen los principios para proteger los recursos naturales, con el fin de no afectar la calidad de vida de las futuras generaciones, este documento se constituye como el corolario de conversaciones entre las diferentes culturas llevadas a cabo en el ámbito mundial durante una década, en las cuales se manifiestan las metas comunes, valores compartidos, esperanzas y aspiraciones de los pueblos de todas las naciones, que tienen la intención de contribuir a la creación de una sociedad global para el siglo XXI en la que prevalezca la justicia social, económica y la armonía ambiental.

La globalización trajo para las empresas esquemas más estructurados para su administración, situación que para algunas representó la quiebra y a otras no les permitió crecer y desarrollarse según sus planes. Hoy, en el siglo XXI, es difícil para las empresas mantenerse en el mercado sin ser afectadas en sus utilidades, el lograr ser rentables y duraderas se ha convertido en el dilema de todas aquellas que no han conseguido que sus procesos económicos se equilibren para obtener más utilidades y así asegurar su crecimiento sostenible.

En el caso de México y quizá de otros países en vías de desarrollo aún no se ha podido diseñar un modelo productivo que apunte al cuidado medioambiental en sus esquemas de producción y de consumo, los cuales están cooperando para que la población 


\section{http://revistainvestigacionacademicasinfrontera.com}

contribuya también a la destrucción ambiental, a la escasez de recursos y la extinción de las especies, hoy por hoy, existen comunidades que han sido destruidas para obtener la riqueza de su subsuelo y garantizar un desarrollo, del cual el beneficio obtenido no se comparte equitativamente y la brecha entre ricos y pobres se hace cada vez más grande, estos efectos nocivos son resultado del comportamiento de los habitantes y de las organizaciones, que les impide tener una concepción más amplia sobre qué constituye el desarrollo sostenible.

De todo lo anterior se desprende la presente investigación, la cual se estructura en tres dimensiones:

1. Dimensión Económica. Se refiere al impacto económico de la organización en sus grupos de interés. En esta dimensión lo primero que hay que considerar son los agentes implicados en el entorno de la organización: mercado, precios, proveedores, derechos de los consumidores, es decir los que soportan los costos o los que disfrutan de los beneficios, el objetivo de la sostenibilidad económica es impulsar el crecimiento en la organización, buscando que las generaciones futuras sean más ricas y que tengan una mejor calidad de vida. El Desarrollo Sustentable debe hacerse en una empresa no como filantropía sino como parte misma de la organización. (Strandberg, 2010)

2. Dimensión social. La dimensión social de la sustentabilidad está relacionada con los impactos de las actividades de una organización en los sistemas sociales en los que opera. La sostenibilidad social pretende que las generaciones futuras tengan más oportunidades que las generaciones anteriores. Pretende sentar las bases para una mejora de nuestra economía mediante incentivos para el progreso de la educación, del conocimiento y de la innovación. (Fernández, 2009)

3. Dimensión medioambiental. La sostenibilidad medioambiental pretende garantizar una gestión responsable y sostenible de los recursos naturales. Quiere legar a las generaciones futuras un entorno natural igual o mejor que el actual, lo 


\section{http://revistainvestigacionacademicasinfrontera.com}

Revista de Investigación

Académica sin Frontera ISSN: 2007-8870

que implica reducir las emisiones contaminantes, una mayor eficiencia en el uso del agua, el suelo y los recursos naturales. (Fernández, 2009)

Estos tres factores se agrupan aplicando los principios de la Responsabilidad social empresarial. Teniendo como objetivo principal analizar el avance de adopción de la Responsabilidad Social en 23 empresas industriales del sur de sonora, el cual nos dará un panorama actual y claro permitiendo detectar las áreas de oportunidad para poder generar cambios positivos dentro de sus actuares y poder adoptar dicho modelo.

\section{Problema de investigación}

En referencia a los antecedentes de la RSE y los factores que han contribuido para que unas empresas la implanten en su gestión administrativa y otras no, por no contar con los medios para desarrollarla o simplemente por desconocimiento, conocer los avances en la implementación de un modelo de responsabilidad social empresarial, constituye la base de la cual partirá la presente investigación realizada en las empresas industriales del sur de sonora, con el propósito fundamental de evaluar si su esquema organizacional corresponde al modelo de empresa socialmente responsable, estudiando hasta qué nivel aplican prácticas de RSE, integrándolas a su actividad empresarial, para el desarrollo de la misma y en beneficio del entorno en que se crearon.

\section{Justificación}

En el sur de Sonora, actualmente se cuenta con 28 empresas industriales dedicadas a producir diferentes artículos que van desde cerveza hasta fabricación de alarmas, entre otros bienes, sin embargo; de las 28 empresas industrias establecidas en el sur de Sonora, se seleccionará una muestra de 23 empresas medianas para llevar a cabo la investigación. Los resultados y beneficios de esta investigación se pondrán a la orden de cada una de las empresas para que conozcan la situación en la que se encuentran y tengan un punto de partida, ya sea para consolidar sus esquemas organizacionales 0 para iniciar esquemas más estructurados que las lleve a obtener la durabilidad, la competitividad que requieran a través de la RSE. 
http://revistainvestigacionacademicasinfrontera.com

ISSN: 2007-8870

\section{Delimitación de la investigación}

El objeto de investigación son las Empresas Industriales del sur de Sonora, las cuales se eligieron a través del padrón de empresas proporcionado por la Cámara Nacional de la Industria de Transformación (CANACINTRA), el cual está integrado por 74 empresas; en este padrón se encuentran empresa comerciales, industriales y de servicio; se eligió una muestra de 23 empresas de clasificación mediana, industriales, la característica para su elección, fue el número de empleados.

\section{Objetivo general}

Evaluar los resultados y definir el nivel de responsabilidad social que presentan las Empresas Industriales del Sur de Sonora.

El propósito fundamental es conocer el nivel de responsabilidad social de estas empresas en las dimensiones ya mencionadas (dimensión económica, social y medioambiental)

\section{Preguntas de investigación}

Lo anterior hace que surjan cuestionamientos acerca de las Empresas industriales del sur de Sonora, conociendo el entorno social, económico y ambiental de esta región, se pueden plantear las siguientes preguntas:

¿Las Empresas Industriales tienen el conocimiento del concepto de RSE en las tres dimensiones (Económico, social y medioambiental) con las que se ha definido? ¿Las Empresas Industriales del sur de Sonora, están en condiciones tanto administrativas como económicas de integrar la responsabilidad social en su gestión? ¿Cuál es actualmente el nivel de responsabilidad social que tiene las Empresas Industriales del Sur de Sonora?

\section{Marco Teórico}

Hechos como la Conferencia de Estocolmo en 1972, el Informe Brundtland en 1987 o el protocolo de Kyoto en 1997; el nacimiento de grupos civiles como Amnistía Internacional, WWF, Greenpeace o Transparencia Internacional; las iniciativas formales 


\section{http://revistainvestigacionacademicasinfrontera.com}

ISSN: 2007-8870

empresariales como AA1000, SA8000, las Directrices de la OCDE o el mismo Pacto Mundial; así como el surgimiento de organizaciones como la Global Reporting Inititative o el Instituto Ethos, son todos, acontecimientos que han servido como bloques para levantar ese modelo de desarrollo, dentro de una construcción social y medioambiental conocida como Responsabilidad Social. (Elizabeth, 2012)

Pero, ¿qué se entiende o se debe entender por responsabilidad Social Empresarial? Para responder a esta pregunta, hay que tener presente que las empresas desarrollan una o varias actividades y para desarrollarlas van implementando procesos diariamente, hasta llegar hacer de estos su modo de hacer las cosas, partiendo de esta libertad que tiene la empresa para accionar, está obligada a tener en cuenta las consecuencias de sus acciones y decisiones asumiendo la responsabilidad de los efectos colaterales de sus quehacer, que afecte a sus diferentes stakeholders. (Barroso, 2008)

La Responsabilidad Social Empresarial (RSE), se puede definir como el compromiso que adquiere una empresa ante sus grupos de interés y la sociedad en general. Se trata de la integración voluntaria por parte de las empresas de las preocupaciones sociales y ambientales en sus operaciones comerciales y en las relaciones con sus interlocutores. Entre otras cosas implica contribuir al desarrollo sostenible, la seguridad, la salud y el bienestar social; respetar y aplicar tanto las normas locales como las internacionales; tener en cuenta los intereses y las expectativas de los grupos de interés, y mantener un comportamiento ético e íntegro, así como la transparencia en su gestión.

Según Olcese, Rodríguez, \& Alfaro, 2008, consideran que el propósito de la empresa responsable y sostenible es perdurar en el tiempo, colaborando en la mejora y progreso del entorno natural y social en que opera. También menciona los cinco fundamentos que constituyen la base del nuevo paradigma de la empresa socialmente responsable y sostenible, siendo estos: La apertura y sensibilidad hacia el entorno, El sentido de 
Año 9. Núm. 24

(Edición especial julio- diciembre 2016)

http://revistainvestigacionacademicasinfrontera.com

ISSN: 2007-8870

comunidad, La capacidad innovadora, La consideración de largo plazo y La creación de valor. (olcese, 2008)

Lo cierto es que las grandes, las pequeñas y medianas empresas, independientemente de la actividad que realicen, deben cumplir con el código de ética de los negocios, que se refiere a la aplicación de la justicia y la equidad, para poder enfrentar situaciones que presentan conflictos éticos entre los stakeholders (Noel, 2008). Hoy más que nunca, las empresas deben desarrollar esa visión ética, debido a la situación en la que se encuentran, que las hace vulnerables a los cambios que la misma economía global exige. Específicamente en Sonora se cuenta con una investigación realizada por el Instituto Tecnológico de Sonora en el que investiga La Red Comunitaria Sonora que contempla una diversidad de empresas en cuanto al número de empleados, el giro productivo y el tipo de sociedad mercantil, haciendo que este estudio sea representativo de las características que tiene la responsabilidad social en el Estado de Sonora. Algunos de los resultados de esta investigación son: de las 31 empresas que participaron, 29 manifestaron un moderado y alto grado de Responsabilidad Social Empresarial (RSE), sin embargo el $50 \%$ no se interesa en la capacitación. Todas manifiestan estar cumpliendo principios éticos pero sólo 11 tienen código de ética. Las empresas carecen de comités de empleados para participar en las decisiones sobre donaciones y aportes. El $50 \%$ de las empresas que pertenecen a la Fundación del Empresariado Sonorense A.C. tienen un programa de desarrollo asociado a los empleados. (Beatriz, 2011)

Muchas empresas consideran que la responsabilidad social se basa en realizar actividades de filantropía, basada en realizar donaciones de dinero o en especie, lo cual no responde a lo que la sociedad espera de ellas, ni garantiza que esas contribuciones voluntarias produzcan resultados sostenibles.

Según lo expuesto, existe gran cantidad de investigaciones sobre la RSE, este representa un tema de gran importancia para las empresas en general y con mayor razón para las empresas industriales del sur de Sonora, debido a la situación que viven actualmente, tal 


\section{http://revistainvestigacionacademicasinfrontera.com}

Revista de Investigación

Académica sin Frontera ISSN: 2007-8870

parecería que no importa el lugar en el que estén operando, sus debilidades son semejantes y requieren de atención para que logren salir adelante. Las empresas del sur de Sonora no podían ser la excepción, por lo que se sugiere una investigación para conocer el grado de responsabilidad social implementado en estas.

\section{DISEÑO METODOLÓGICO}

Tipo de investigación. La investigación a realizarse será de tipo Descriptiva, ya que busca especificar propiedades, características y rasgos importantes de cualquier fenómeno que se analice, lo que se pretende es describir el porqué de un fenómeno y en qué condiciones se da este, o por qué dos o más variables están relacionadas.

No experimental, ya que no se tiene control sobre las variables independientes porque ya ocurrieron los hechos o porque son intrínsecamente manipulables, la variación de estas se logrará no por manipulación directa sino por medio de la selección de las unidades de análisis en las que la variable estudiada tiene presencia, como puede ser el desempeño de las empresas respecto a la variable.

Transversal, ya que se encarga de recolectar datos en un solo momento y en un tiempo único (se utilizó este tipo de investigación ya que se recolectó datos mediante cuestionarios en un mismo periodo de tiempo).

En esta etapa se procederá a la recopilación y análisis de datos relacionados con el tema de investigación. Llevando a cabo las siguientes actividades:

Escala Likert: Las dimensiones que se determinaron para conocer el grado de Responsabilidad Social Empresarial son las económicas, sociales y medioambientales, y se medirá en una encuesta elaborada en una escala Likert con los siguientes ítems: 
Año 9. Núm. 24 (Edición especial julio- diciembre 2016)

Revista de Investigación

Académica sin Frontera

ISSN: 2007-8870

\section{http://revistainvestigacionacademicasinfrontera.com}

\section{INSTRUMENTO}

Se agradece su participación para conocer sus acciones encaminadas hacia la Responsabilidad Social empresarial. Por favor complete el siguiente cuestionario lo más honestamente posible, con la garantía de que sus respuestas y opiniones serán tratadas de forma global y anónima.

Instrucciones: Considere la siguiente escala para responder:

\begin{tabular}{|c|c|c|c|c|}
\hline $\mathbf{1}$ & $\mathbf{2}$ & $\mathbf{3}$ & $\mathbf{4}$ & $\mathbf{5}$ \\
\hline $\begin{array}{c}\text { Totalmente } \\
\text { en } \\
\text { desacuerdo }\end{array}$ & $\begin{array}{c}\text { En } \\
\text { desacuerdo }\end{array}$ & $\begin{array}{c}\text { Ni de } \\
\text { acuerdo ni } \\
\text { en } \\
\text { desacuerdo }\end{array}$ & De acuerdo & $\begin{array}{c}\text { Totalmente } \\
\text { de acuerdo }\end{array}$ \\
\hline
\end{tabular}

De acuerdo a su experiencia vivida valore de forma precisa entre 1 ("Totalmente en desacuerdo") y 5 ("Totalmente de acuerdo") su percepción sobre las siguientes enunciaciones acerca de la Responsabilidad Social de las industrias:

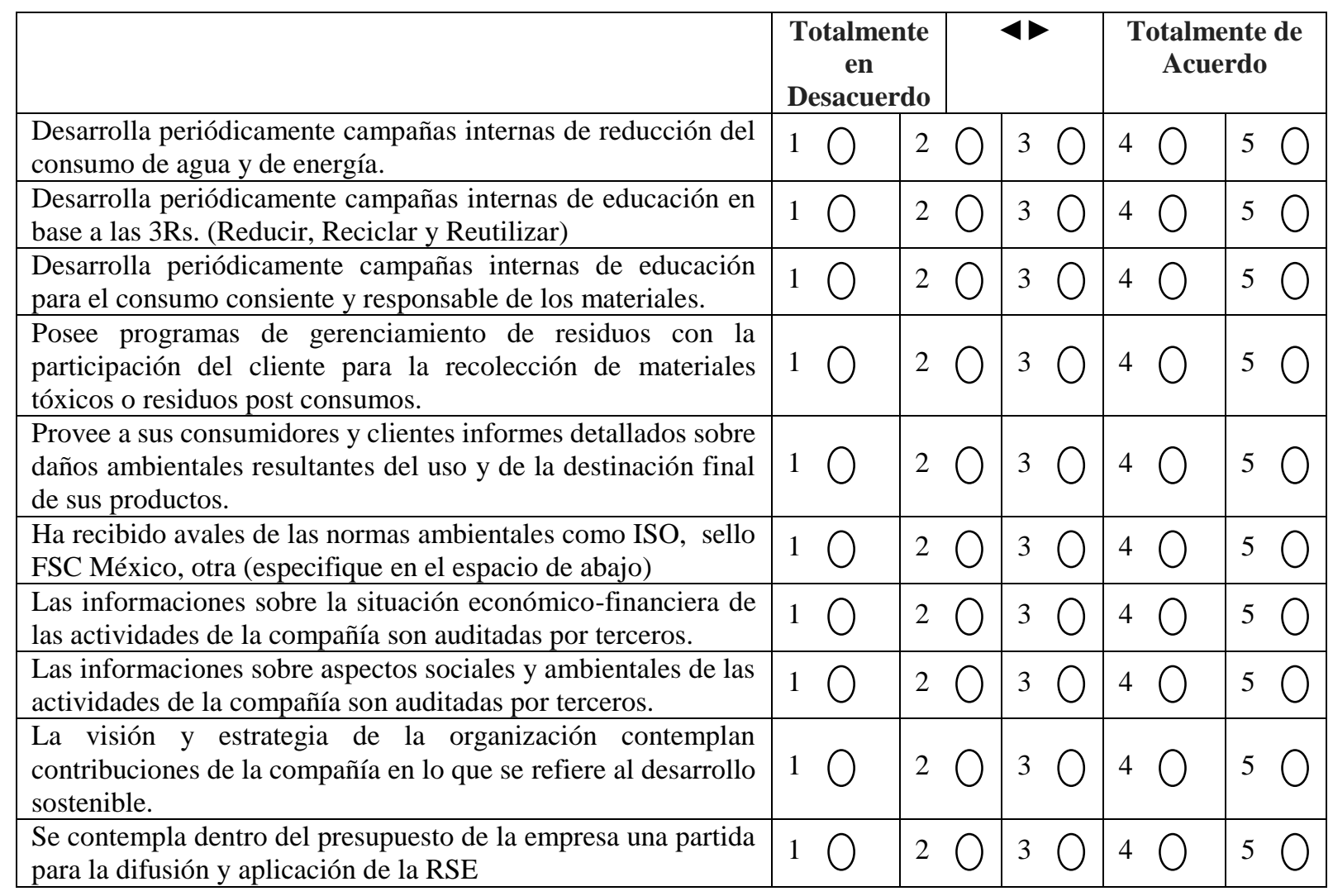

Preguntas de clasificación general:

\begin{tabular}{|l|l|l|l|l|l|l|l|l|}
\hline Antigüedad: & 1. & De 1 a 3 años & 2 . & Entre 4 y 10 & 3. Entre 11 y 15 & 4. Entre 16 y 20 & 5. & Más de20 años
\end{tabular}

Número de empleados: 1 . Hasta 5 2. 6 . 6 a 10 3. 11 a 50 4. 51 a 100 5. Más de 100

Tipo de certificación obtenida 1. 3. 
Nivel de estudios de su Gerente/Director:

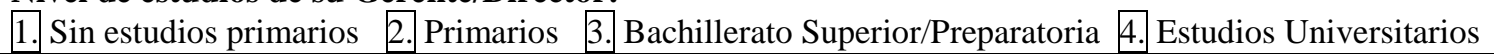

Clasificación de la empresa:

1. Manufacturera

2. Extractiva

Ciudad:

Estado:

\section{RESULTADOS POR DIMENSIÓN}

\section{DIMENSIÓN AMBIENTAL}

Análisis de la información en relación a la DIMENSIÓN AMBIENTAL la cual abarca las preguntas número $1,2,3,4$ y 6 donde se conoce el nivel que se manifiesta según la respuesta de las encuestas.

Siendo cuatro preguntas con un valor máximo de 5 puntos por 5 preguntas es $=25$ puntos como puntación máxima.

El análisis muestra que solo 1 empresa alcanzo la puntuación máxima, 2 empresas con 24 puntos, tres con 23, 5 empresas con 22 puntos, 2 empresas con 21 , una con 20 puntos, una con 19 puntos, 5 con 18 y 3 con 17 puntos.

El resultado nos indica que todas las empresas sí aplican campañas internas de consumo de agua y energía; el 97\% de las estas, manifiestan que si desarrollan campañas de educación en base a las 3rs; de la misma manera el porcentaje sobre la aplicación consiente y responsable de los materiales es del 95\%; respecto al gerenciamiento de residuos el $82.6 \%$ manifiesta que sí posee dichos programas; mientras que de las 23 empresas investigadas solo el $62.5 \%$ manifestaron que cuentan con algún tipo de aval sobre normas ambientales, destacando entre ellas Empresa Limpia o (PNAA) Programa Nacional de Auditoría Ambiental y Sistema de Gestión de Calidad (ISO 9001); indicando que la responsabilidad en relación a la dimensión ambiental alcanza un nivel alto, concluyendo que lo más importante es buscar por parte de las empresas las certificaciones que avalen la responsabilidad ambiental, con ello se lograría mejorar en todo los aspecto los diferentes puntos que incluye la dimensión ambiental. 
Año 9. Núm. 24 (Edición especial julio- diciembre 2016)

http://revistainvestigacionacademicasinfrontera.com

Revista de Investigación

Académica sin Frontera ISSN: 2007-8870

\section{DIMENSIÓN SOCIAL}

Análisis de la información en relación a la DIMENSIÓN SOCIAL la cual abarca las preguntas número 5,8 y 9 , los resultados que arrojan las encuestas son:

Siendo tres preguntas con un valor máximo de 5 puntos por 3 preguntas es $=15$ puntos como puntación máxima.

El análisis muestra que 7 empresas alcanzaron la puntuación máxima de 15 puntos, 5 empresas con 14 puntos, tres con 13, 2 empresas con 12 puntos, 3 empresas con 11, dos con 9 puntos y una con 8 puntos.

El resultado en relación a los informes sobre daños ambientales en relación a la destinación final sobre su productos, el porcentaje alcanzo un $78.3 \%$; mientras que la información sobre aspectos sociales y ambientales de las actividades de la compañía son auditadas por terceros, refleja un $73.9 \%$; en lo relacionado a que si la empresa contempla en la visión y estrategia organizacional el concepto de desarrollo sustentable, este alcanzo un $95.7 \%$. Indicando que la responsabilidad en relación a la dimensión social alcanza un nivel de medio a alto, manifestando que las empresas hoy en día si tiene un mayor interés de mejorar o contribuir al mejoramiento del lugar donde están establecidas, sin dejar de percibir que aún se puede mejorar mucho lo que se hace.

\section{DIMENSIÓN ECONÓMICA}

Análisis de la información en relación a la DIMENSIÓN ECONÓMICA la cual abarca las preguntas número 7 y 10, los resultados que arrojan las encuestas son:

Siendo dos preguntas con un valor máximo de 5 puntos por 2 preguntas es $=10$ puntos como puntación máxima.

El análisis muestra que 13 empresas alcanzaron la puntuación máxima de 10 puntos, 5 empresas con 9 puntos, una con 8, 2 empresas con 7 puntos, una empresa con 6 puntos, y una con 5 puntos.

el resultado sobre la dimensión económica en relación a que si las empresas son auditadas por terceros sobre la situación económica - financiera alcanza un 100\%; sobre 
Año 9. Núm. 24 (Edición especial julio- diciembre 2016)

\section{http://revistainvestigacionacademicasinfrontera.com}

la pregunta donde se menciona si la empresa contempla una partida para la difusión y aplicación de la RSE suma un porcentaje del $78.2 \%$. De las 23 empresas encuestadas 18 tiene una puntuación alta, lo cual nos indica que respecto a la dimensión económica si se cumple en relación a las auditorias financieras, considerando que es una obligación respecto a la ley, pero sobre la difusión y aplicación de la RSE, si hay mucho que hacer.

\section{RESULTADOS POR PREGUNTA}

Cuadro 1. (Pregunta 1) ¿Desarrolla periódicamente campañas internas de reducción del consumo de

\begin{tabular}{|l|c|c|}
\hline \multicolumn{3}{|c|}{ agua y de energía? } \\
\hline DiMENSIÓN AMBIENTAL \\
\hline De acuerdo & Num. Respuestas & Porcentaje \\
\hline Totalmente de acuerdo & 10 & $43,5 \%$ \\
\hline Total & 13 & $56,5 \%$ \\
\hline
\end{tabular}

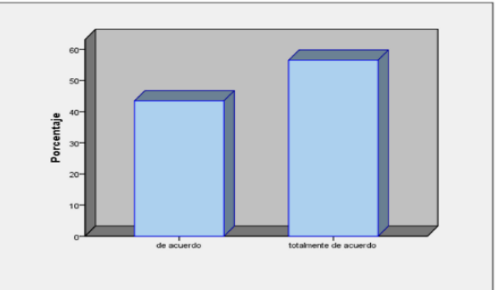

Cuadro 2. (Pregunta 2) ¿Desarrolla periódicamente campañas internas de educación con base en las 3Rs. (Reducir, Reciclar y Reutilizar)?

\begin{tabular}{|c|c|c|}
\hline \multicolumn{3}{|c|}{ DIMENSIÓN AMBIENTAL } \\
\hline Repuestas & Num. Respuestas & Porcentaje \\
\hline Ni de acuerdo ni en desacuerdo & 3 & $13,0 \%$ \\
\hline De acuerdo & 10 & $43,5 \%$ \\
\hline Totalmente de acuerdo & 10 & $43,5 \%$ \\
\hline Total & 23 & $100,0 \%$ \\
\hline
\end{tabular}

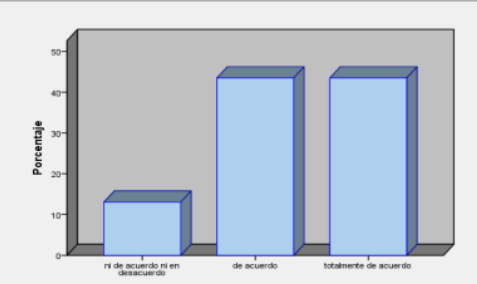

Cuadro 3. (Pregunta 3) ¿Desarrolla periódicamente campañas internas de educación para el consumo consiente y responsable de los materiales?

\begin{tabular}{|l|c|c|}
\hline \multicolumn{3}{|c|}{ DIMENSIÓN AMBIENTAL } \\
\hline \multicolumn{1}{|c|}{ Repuestas } & $\begin{array}{c}\text { Numero de } \\
\text { Respuestas }\end{array}$ & Porcentaje \\
\hline Ni de acuerdo ni en desacuerdo & 1 & $4,3 \%$ \\
\hline De acuerdo & 9 & $39,1 \%$ \\
\hline Totalmente de acuerdo & 13 & $56,5 \%$ \\
\hline Total & $\mathbf{2 3}$ & $\mathbf{1 0 0 , 0} \%$ \\
\hline
\end{tabular}

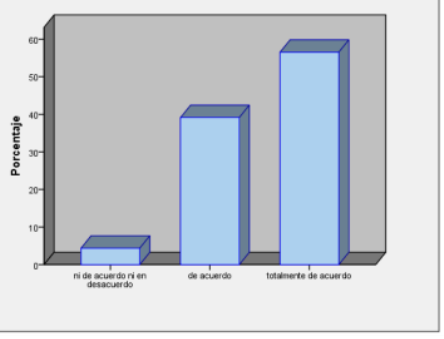


Año 9. Núm. 24 (Edición especial julio- diciembre 2016)

\section{http://revistainvestigacionacademicasinfrontera.com}

Cuadro 4. (Pregunta 4) ¿Posee programas de gerenciamiento de residuos con la participación del cliente para la recolección de materiales tóxicos o residuos post consumos?

\begin{tabular}{|l|c|c|}
\hline \multicolumn{3}{|c|}{ DIMENSIÓN AMBIENTAL } \\
\hline Repuestas & Num Respuestas & Porcentaje \\
\hline Totalmente en desacuerdo & 2 & $8,7 \%$ \\
\hline En desacuerdo & 1 & $4,3 \%$ \\
\hline Ni de acuerdo ni en desacuerdo & 1 & $4,3 \%$ \\
\hline De acuerdo & 5 & $21,7 \%$ \\
\hline Totalmente de acuerdo & 14 & $60,9 \%$ \\
\hline Total & $\mathbf{2 3}$ & $\mathbf{1 0 0 , 0 \%}$ \\
\hline
\end{tabular}

Cuadro 5. (Pregunta 6) ¿Ha recibido avales de las normas ambientales como ISO, sello FSC México u otras?

\begin{tabular}{|c|c|c|}
\hline \multicolumn{3}{|c|}{ DIMENSIÓN AMBIENTAL } \\
\hline Repuestas & Num Respuestas & Porcentaje \\
\hline Totalmente en desacuerdo & 6 & $26,1 \%$ \\
\hline Ni de acuerdo ni en desacuerdo & 2 & $8,7 \%$ \\
\hline De acuerdo & 4 & $17,4 \%$ \\
\hline Totalmente de acuerdo & 11 & $47,8 \%$ \\
\hline Total & 23 & $100,0 \%$ \\
\hline
\end{tabular}

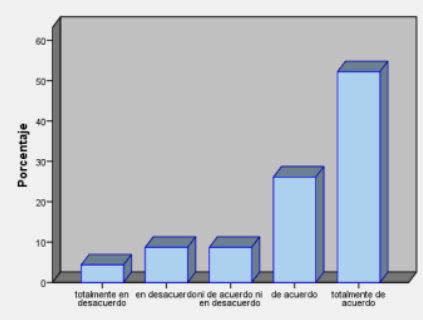

Cuadro 6. (Pregunta 5) ¿Provee a sus consumidores y clientes informes detallados sobre daños ambientales resultantes del uso y de la destinación final de sus productos?

\begin{tabular}{|l|c|c|}
\hline \multicolumn{3}{|c|}{ DIMENSIÓN SOCIAL } \\
\hline \multicolumn{1}{|c|}{ Repuestas } & $\begin{array}{c}\text { Numero de } \\
\text { Respuestas }\end{array}$ & Porcentaje \\
\hline Totalmente en desacuerdo & 1 & $4,3 \%$ \\
\hline En desacuerdo & 2 & $8,7 \%$ \\
\hline Ni de acuerdo ni en desacuerdo & 2 & $8,7 \%$ \\
\hline De acuerdo & 6 & $26,1 \%$ \\
\hline Totalmente de acuerdo & 12 & $52,2 \%$ \\
\hline Total & $\mathbf{2 3}$ & $\mathbf{1 0 0 , 0 \%}$ \\
\hline
\end{tabular}

Cuadro 7. (Pregunta 8) ¿Las informaciones sobre aspectos sociales y ambientales de las actividades de la compañía son auditadas por terceros? 
Año 9. Núm. 24 (Edición especial julio- diciembre 2016)

\section{http://revistainvestigacionacademicasinfrontera.com}

\begin{tabular}{|l|c|c|}
\hline \multicolumn{3}{|c|}{ DIMENSIÓN SOCIAL } \\
\hline Repuestas & Num. Respuestas & Porcentaje \\
\hline Totalmente en desacuerdo & 4 & $17,4 \%$ \\
\hline Ni de acuerdo ni en desacuerdo & 2 & $8,7 \%$ \\
\hline De acuerdo & 2 & $8,7 \%$ \\
\hline Totalmente de acuerdo & 15 & $65,2 \%$ \\
\hline Total & $\mathbf{2 3}$ & $\mathbf{1 0 0 , 0} \%$ \\
\hline
\end{tabular}

Cuadro 8. (Pregunta 9) ¿La visión y estrategia de la organización contemplan contribuciones de la compañía en lo que se refiere al desarrollo sostenible?

\begin{tabular}{|l|c|c|}
\hline \multicolumn{3}{|c|}{ DIMENSIÓN SOCIAL } \\
\hline Repuestas & Num. Respuestas & Porcentaje \\
\hline Ni de acuerdo ni en desacuerdo & 1 & $4,3 \%$ \\
\hline De acuerdo & 4 & $17,4 \%$ \\
\hline Totalmente de acuerdo & 18 & $78,3 \%$ \\
\hline Total & $\mathbf{2 3}$ & $\mathbf{1 0 0 , 0} \%$ \\
\hline
\end{tabular}

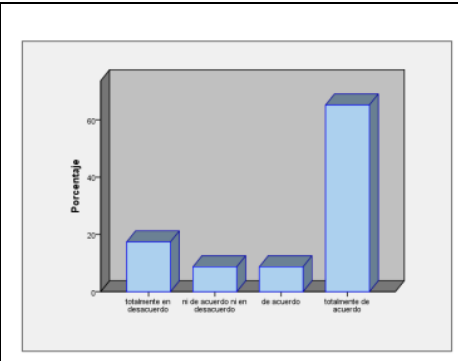

Cuadro 9. (Pregunta 7) ¿Las informaciones sobre la situación económico-financiera de las actividades de la compañía son auditadas por terceros?

\begin{tabular}{|l|c|c|}
\hline \multicolumn{3}{|c|}{ DIMENSIÓN ECONOMICA } \\
\hline Repuestas & Num. Respuestas & Porcentaje \\
\hline De acuerdo & 4 & $17,4 \%$ \\
\hline Totalmente de acuerdo & 19 & $82,6 \%$ \\
\hline Total & $\mathbf{2 3}$ & $\mathbf{1 0 0 , 0 \%}$ \\
\hline
\end{tabular}

Cuadro 10. (Pregunta 10) ¿Se contempla dentro del presupuesto de la empresa una partida para la difusión y aplicación de la RSE? 
Año 9. Núm. 24 (Edición especial julio- diciembre 2016)

http://revistainvestigacionacademicasinfrontera.com

\begin{tabular}{||l|c|c|}
\hline \multicolumn{2}{|c|}{ DIMENSIÓN SOCIAL } \\
\hline \multicolumn{1}{|c|}{ Repuestas } & Num Respuestas & Porcentaje \\
\hline Totalmente en desacuerdo & 3 & $13,0 \%$ \\
\hline Ni de acuerdo ni en desacuerdo & 2 & $8,7 \%$ \\
\hline De acuerdo & 3 & $13,0 \%$ \\
\hline Totalmente de acuerdo & 15 & $65,2 \%$ \\
\hline Total & $\mathbf{2 3}$ & $\mathbf{1 0 0 , 0} \%$ \\
\hline
\end{tabular}

\section{Conclusiones}

Después de analizar y procesar los resultados de la investigación, los cuales se obtuvieron a través del programa estadístico SPSS versión 21.0 se llega a las siguientes conclusiones:

1. Las empresas Industriales del sur de Sonora sí conocen el concepto de Responsabilidad Social Empresarial (RSE), más sin embargo no se aplica en su totalidad, ya que existen algunas empresas que no realizan las actividades concernientes a la normatividad que exige la misma.

2. Por otro lado un $95.6 \%$ de las empresas analizadas están desarrollando periódicamente campañas internas de educación para el consumo consiente y responsable de los materiales, así como también campañas internas con base en las 3Rs. (Reducir, Reciclar y Reutilizar), así como reducción del consumo de agua y de energía.

3. El $95.7 \%$ de las empresas del sur de Sonora están en condiciones de integrar la responsabilidad social en su gestión, ya que la contemplan en su visión y estrategia organizacional, además se efectúan auditorías ambientales en un $73.9 \%$ de las empresas encuestadas. Otro aspecto importante es la labor que hacen las empresas al proporcionar información a sus clientes sobre la RSE, por ejemplo, se puede 


\section{http://revistainvestigacionacademicasinfrontera.com}

ISSN: 2007-8870

resaltar que el $78.3 \%$ de las empresas les proporcionan información detallada sobre daños ambientales resultantes del uso y de la destinación final de sus productos, aunque lo anterior no es suficiente ya que el $21.7 \%$ aún no posee programas de gerenciamiento de residuos con la participación del cliente para la recolección de materiales tóxicos o residuos post consumos.

4. En relación al nivel de responsabilidad social, el $65.2 \%$ de las empresas industriales del sur de Sonora, cuentan con algún aval de normas ambientales como lo son ISO, Empresa Limpia o PNAA, sello FSC México, entre otras, esto nos indica que cada vez más empresas se están preocupando y ocupando de contar con éste tipo de certificación, aunque no podemos decir que ya estamos satisfechos totalmente con los resultados porque la contaminación y la mala utilización de los desechos se sigue dando por algunas empresas, todavía existen empresarios que prefieren evadir esas responsabilidades y seguir contaminando, por ejemplo, en la Ciudad de Navojoa se tiene un bonito Río denominado "Río Mayo" el cual todos sabemos que esas aguas están contaminadas, debido a que muchas empresas arrojan desechos tóxicos en él y aunque las mismas autoridades anuncian que está contaminado, no se hace nada para evitarlo..

\section{Recomendaciones}

Los resultados obtenidos son aceptables y reflejan el trabajo y compromiso de las empresas por cumplir con las normas de responsabilidad social, más sin embargo se debe de establecer un programa de difusión en el cual se expliquen los beneficios de ser una empresa socialmente responsable, enfocándose al 34.8\% de las empresas que aún no cuentan con un aval de calidad en dicha materia.

Así mismo se recomienda aumentar los programas de capacitación para los empleados en función de reducir, reutilizar y reciclar, manteniendo una educación constante y continua, para que de esta manera puedan replicar los métodos utilizados dentro de las 
http://revistainvestigacionacademicasinfrontera.com

Revista de Investigación

Académica sin Frontera ISSN: 2007-8870

empresas en su vida diaria y contribuir de esta manera a la mejora del medio ambiente, no solo dentro de la organización sino también dentro de la comunidad.

Es importante que la certificación en normas ambientales sea un requisito obligatorio, dando seguimiento a través de visitas sorpresa para cerciorarse de que siguen cumpliendo, ya que en muchos de los casos solamente se cumple con los requerimientos durante la visita de los que evalúan y posteriormente se desatienden o bajan la guardia, cumpliendo a medias.

\section{Bibliografía}

Barroso, T. (2008). La Responsabilidad Social empresarial. Un estudio de cuarrenta empresas de la ciudad de Mérida, Yucatán. Contabilidad y Administración No. 226.

Beatriz, O. S. (2011). Responsabilidad Social Empresarial: Un Modelo Integral para Sonora. Cd. Obregon: Instituto Tecnologico de Sonora.

Elizabeth, L. R. (2012). una mirada a la Responsabilidad Social en México. Letras y Numeros.

Fernández, G. R. (2009). La Dimensión Económica del desarrollo Sostenible. Editorial Club Universitario.

Noel, R. P. (2008). Contabilidad Administrativa. Mexico D.F.: McGraw-Hill.

olcese, R. y. (2008). Mnual de la Empresa Responsable y sostenible. Madrid: Mcgraw-Hill/Interamericana de España.

Strandberg, L. (marzo de 2010). http://www.iese.edu/es/files/Cuaderno\%20No\%2010_tcm5-61597.pdf. 